\title{
Visions littéraires des paysages de la bonification en Italie
}

Federica Letizia Cavallo

\section{Citer ce document / Cite this document :}

Cavallo Federica Letizia. Visions littéraires des paysages de la bonification en Italie. In: Le Globe. Revue genevoise de géographie, tome 156, 2016. Italie. Paysage et identité. pp. 111-134;

doi : $10.3406 /$ globe.2016.7398

http://www.persee.fr/doc/globe_0398-3412_2016_num_156_1_7398

Document généré le 16/02/2017 


\title{
Résumé
}

L'article analyse, dans une clé géocritique, les représentations de l'espace et du paysage des bonifications en Italie, telles qu'elles émergent de quelques oeuvres littéraires. On observera en particulier trois phases du regard littéraire sur le paysage des bonifications et certains thèmes qui les traversent. D'une phase classique, centrée sur l'éloge indirect des bonifications en tant que dépassement des marais, on arrive à une phase de modernisation fasciste, qui voit dans les bonifications la construction d'un paysage à l'image du régime (ordre, géométrie, machinisme). La troisième phase, contemporaine, reconsidère les paysages de la bonification dans le sens d'une humanisation, d'un vécu et d'un sens du lieu retrouvé.

\begin{abstract}
This paper analyses, from a geo-critical viewpoint, the representations of Italian land reclamation space and landscape, as reflected in some literary works. In particular, three phases of the literary gaze on land reclamation landscape are considered, as well as some themes running through them. From a classical phase, centred on the indirect eulogy of land reclamation as a mean to overcome the swamp, we get to the phase of fascist modernization, which reads in land reclamation the construction of an efficient landscape guided by order, geometry and mechanic. The third phase, the contemporary one, sees a reconsideration of land reclamation landscape, marked by humanization, lived experience and a renewed sense of place.
\end{abstract}




\title{
VISIONS LITTERAIRES DES PAYSAGES DE LA BONIFICATION EN ITALIE
}

\author{
Federica Letizia CAVALLO \\ Université Ca' Foscari de Venise
}

Résumé : L'article analyse, dans une clé géocritique, les représentations de l'espace et du paysage des bonifications en Italie, telles qu'elles émergent de quelques oeuvres littéraires. On observera en particulier trois phases du regard littéraire sur le paysage des bonifications et certains thèmes qui les traversent. D'une phase classique, centrée sur l'éloge indirect des bonifications en tant que dépassement des marais, on arrive à une phase de modernisation fasciste, qui voit dans les bonifications la construction d'un paysage à l'image du régime (ordre, géométrie, machinisme). La troisième phase, contemporaine, reconsidère les paysages de la bonification dans le sens d'une humanisation, d'un vécu et d'un sens du lieu retrouvé.

Mots-clés : Bonification, paysage, Italie, géographie littéraire, Vincenzo Monti, Corrado Alvaro, Antonio Pennacchi.

\begin{abstract}
This paper analyses, from a geo-critical viewpoint, the representations of Italian land reclamation space and landscape, as reflected in some literary works. In particular, three phases of the literary gaze on land reclamation landscape are considered, as well as some themes running through them. From a classical phase, centred on the indirect eulogy of land reclamation as a mean to overcome the swamp, we get to the phase of fascist modernization, which reads in land reclamation the construction of an efficient landscape guided by order, geometry and mechanic. The third phase, the contemporary one, sees a reconsideration of land reclamation landscape, marked by humanization, lived experience and a renewed sense of place.
\end{abstract}

Keywords : Land Reclamation, Landscape, Italy, Literary Geography, Vincenzo Monti, Corrado Alvaro, Antonio Pennacchi.

\section{Les paysages de la bonification en Italie et l'analyse géolittéraire}

Même si les installations hydrauliques font partie de sa tradition depuis des siècles, la péninsule italienne se présente à l'époque contemporaine avec de vastes zones de plaines, notamment celles côtières et de l'arrièrepays, toujours caractérisées par la présence de marais.

Entre le $\mathrm{XVIII}^{\mathrm{e}}$ et le $\mathrm{XIV}^{\mathrm{e}}$ s. déjà, avec un accroissement durant la période post-unitaire, on procède à des interventions modernes de 
bonification des terres, sous forme d'installations hydrauliques, pour des raisons sociosanitaires et de production économique ; ce fut l'un des points stratégiques des politiques publiques italiennes. Tendance qui se poursuit dans les années 1900 et qui s'accentue particulièrement pendant les vingt années du fascisme (Stampacchia, 1983 et 2000 ; Novello, 2003). Le lien entre fascisme et bonification est manifeste aussi grâce à l'amplification de la propagande que le régime réservait aux interventions hydrauliques et aux aménagements agricoles. De telles interventions aspiraient à des aménagements du territoire ex nihilo et ont donc abouti à des changements importants du paysage.

Le mélange de terre et d'eau conférait aux paysages humides le caractère typique des milieux amphibiens, où les pratiques et les expériences des populations étaient centrées sur des techniques d'anthropisation, généralement résistantes et adaptées à l'environnement. En revanche, grâce aux bonifications hydrauliques mécaniques, on délimite des paysages agraires centrés sur le contrôle des eaux, aussi bien en matière de drainage que d'irrigation (Cavallo, 2011).

Ces paysages sont le fruit d'un projet technique d'ingénierie hydraulique mis en œuvre grâce à un déploiement important de travail et de capitaux (publics et privés), ainsi qu'à un processus d'excavation et de transfert de terre. Un tel projet était soutenu par la volonté de moderniser les zones rurales, dont la sécurité hydraulique était perçue comme la condition sine qua non à la maximisation de la productivité, au point de permettre un système d'installation plus stable, peuplé et planifié, par rapport à ceux que l'on rencontre en général dans les zones humides : maisons et centres ruraux et, parfois, villes nouvelles (Mioni, 1976).

A partir du courant d'études réunissant géographie et littérature (Lando, 1993 ; Chevalier, 1993 et 2001 ; Brosseau, 1996 ; De Fanis, 2001 ; Westphal, 2007 ; Collot, 2014), cette contribution propose d'analyser l'image du paysage de bonification tel qu'il apparaît dans certaines œuvres italiennes (en prose ou en vers), qu'elles soient "historiques" (datant des débuts de l'époque contemporaine), ou actuelles. Trois œuvres sont particulièrement prises en considération, car elles symbolisent trois visions successives du paysage de bonification. Nous pourrions parler, respectivement, d'une phase classique, d'une phase de modernisation fasciste et d'une phase plus étroitement contemporaine (qui ne peut faire abstraction du dialogue, explicite ou implicite, avec les représentations

LE GLOBE - TOME 156 - 2016 
fascistes des terres de bonification). Les trois œuvres en question sont : le poème La Feroniade de Vincenzo Monti (1784), le compte-rendu romancé Terra Nuova. Prima cronaca dell'Agro Pontino de Corrado Alvaro (1934) et le roman Canal Mussolini d'Antonio Pennacchi (2010). Grâce aux répercussions remarquables qu'elles ont obtenues (chacune à son époque) et à leur capacité de médiation marquée entre l'aspect physique et symbolique des paysages de bonification (Pocock, 1981), ces œuvres représentent, pour ainsi dire, le fil conducteur de notre contribution. Les œuvres citées sont accompagnées d'autres références littéraires qui font office d'ébauche : le reportage Entretiens avec Mussolini d'Emil Ludwig (1932), le discours Estrazione sistematica di nuovi splendori e nuove musiche dai tecnicismi de Filippo Tommaso Marinetti (1940), le récit/roman Amado mio de Pier Paolo Pasolini (1948), l'autobiographie Mi richordo anchora di Pietro Ghizzardi (1967), la poésie I fosseti et le roman La lodola mattiniera (1977), tous deux de Romano Pascutto, et le récit " $\Delta$ " de Dario Voltolini (2004). L'approche abordée ici est essentiellement de type géocritique (Westphal, 2007 ; Collot, 2014), c'est-à-dire qu'elle cherche à identifier et analyser les représentations de l'espace et du paysage de bonification dans les œuvres littéraires citées, mais aussi à étudier les interactions entre les espaces de bonification et la littérature, si nous partons du principe que "l'environnement humain est une construction culturelle autant qu'économique, et la littérature contribue à cette construction" (Collot, 2014).

Dans cette perspective, les représentations et les sens attribués aux paysages de bonification dans les textes littéraires évoquent, d'une part, les idéaux et la culture liés aux territoires dont les auteurs s'érigent en porte-parole. De cette manière, ces derniers ne "décrivent" pas le paysage de bonification, mais l'écrivent" (conformément au fait historico-social et au climat culturel auquel ils participent). D'autre part, les connotations que les auteurs, avec leurs propres codes, attribuent au paysage de bonification finissent par devenir, par le biais de leur influence et/ou de leur efficacité géopoétique, des filtres permettant à d'autres individus (et, à la limite, à une société dans son intégralité) de regarder ces paysages, d'en saisir le caractère "marquant" car représentés comme tels, d'en fixer certains canons esthétiques et de mettre en relief des sens symboliques déterminés. 


\section{Les origines de l'éloge littéraire du paysage de bonification : la réhabilitation des marais}

Les paysages de bonification hydraulique ne répondent pas aux canons pittoresques du "beau paysage" et ne font certainement pas partie des paysages italiens les plus reconnus en matière d'iconographie. Il existe néanmoins un courant littéraire consacré à ce sujet. Datant en grande partie de la période fasciste, dû notamment à l'élan provenant du Sindacato Fascista Autori e Scrittori, ce courant repose sur des précédents significatifs. Parmi ces précédents, le poème de Vincenzo Monti intitulé La Feroniade (1784, puis publié inachevé en 1832). Composée pour l'occasion, l'œuvre rend hommage à la tentative de bonification des marais pontins effectuée par le pape Pie VI (Giovanni Braschi) ${ }^{1}$ entre les années 1770 et 1790, en transfigurant les essais de l'époque romaine, des consuls Appius et Cethegus et des empereurs Auguste et Trajan en narration mythologique, typique des tendances néoclassiques. Le poète imagine qu'autrefois, dans une campagne peuplée décrite sur un ton géorgique vers Terracina, demeurait la nymphe Féronie, aimée de Jupiter et élevée pour cette raison au rang de déesse. Mais Junon, aveuglée par sa jalousie, avait déchainé la colère des fleuves Ufente, Astura et Ninfeo sur ces terres, ce qui en provoqua l'inondation (Monti, 1839:476-492) :

Déjà tout le beau règne de Feronia avait été transformé en horrible marais noir qui paraissait une mer (...)

l'horrible marais

battu par des vents frais et houleux

tandis que les rayons du soleil en direction du coucher couraient vermeils sur le flot instable ; du soleil qui paraissait s'enfuir piteux de cette vue impie (...)

la pontine

vallée transformée en horrible mer

Les attributs du marais, repris tout au long du poème, sont aussi canoniques qu'explicitement négatifs : il est horrible, sombre ("atra") et étendu à perte de vue, au point de s'apparenter à la mer. Néanmoins, Jupiter consola Féronie en lui divulguant une prophétie : la remise en état du marais sera assurée ; tout d'abord par certains empereurs romains, dont

LE GLOBE - TOME 156 - 2016 
les interventions ne seront pas définitives, et, ensuite, par le pape auquel la Feroniade est dédiée (Monti, 1839:506-507) :

\section{(...) Appius, qui tracera}

au milieu des troubles marais pontins

la reine des voies [la Via Appia]. Puis Cethegus

ensuite le puissant et heureux Auguste (...)

le pieu Trajan

qui, ayant dompté des armes Asie et Europe,

avec la raison domptera ton marais $(. .$.

l'ouvrage

immortel, glorieux et non fini,

réserve son destin à un plus grand héros

qui sera nommé Pius par les gens et de ce nom

$\mathrm{VI}^{\mathrm{e}}$ il sera $(\ldots)$

Par une gradation symbolique, selon laquelle les routes qui traversent le marais sont d'abord établies pour ensuite 1'"apprivoiser", notons l'aspect héroïque des auteurs de la bonification, qui trouve son apogée en la figure de Pie VI. D'ailleurs, le nom du canal d'écoulement qui retourne vers la Via Appia s'appelle encore aujourd'hui "linea Pia" en hommage au pape. L'éloge de Monti est implicite et se déroule sous forme d'antithèses : le paysage de bonification est loué en tant que réhabilitation du marais. Les formules plutôt classiques n'insistent pas sur les résultats de la bonification, mais anticipent l'époque de son éloge explicite par rapport à l'image mortifère du marais.

Eloge de la construction d'un paysage "moderne" (et nostalgie de l'ancien paysage ?)

C'est évidemment pendant les vingt années du fascisme que les éloges littéraires du paysage de bonification se multiplient et se gonflent de la rhétorique du régime. Les marais pontins sont naturellement le décor principal, mais les bonifications des basses vallées vénéto-frioulanes et émilien-romagnoles sont également fréquentées par les auteurs de l'époque fasciste 2 .

En 1934 paraît Terra Nuova, Prima cronaca dell'Agro Pontino de Corrado Alvaro, écrit pour servir de modèle à l'Istituto Nazionale di Cultura Fascista. Il s'agit d'une chronique romancée écrite à la première personne, dont l'objectif et le style sembleraient ouvertement élogieux,

LE GLOBE - TOME 156 - 2016 
conformes à la tendance de la propagande. En vérité, l'œuvre laisse entendre des réflexions plus complexes sur les paysages "naturels", les interventions qui en bouleversent la structure et les destins des habitants des marais ou colons de la bonification, impliqués dans des évènements qui les dépassent et destinés à s'adapter au changement radical de leur lieu de vie. L'œuvre restitue avant tout la représentation progressive d'un "espace en construction" aussi bien rural qu'urbain. La chronique débute en effet par la naissance symbolique de la ville de Sabaudia :

L'ingénieur marchait derrière les ouvriers... L'un d'eux, le dernier, portait une planche en bois vernie de blanc, sur laquelle était écrit en majuscules SABAUDIA. [...] L'ingénieur pointa son doigt. L'homme avec à la planche s'arrêta entre les deux rangées d'ouvriers, planta le piquet sur lequel était clouée la planche... Ce nom s'imposait comme le premier mot écrit dans ce monde élémentaire d'arbres et d'eaux" (Alvaro, 2008:11).

Si les villes pontines renvoient à un acte unique de fondation, habité par une aura mythique, la bonification agraire est, en revanche, représentée par un paysage en construction qui trouve son élément fondamental dans la régulation des cours d'eau :

Les grandes pluies de ce mois de novembre (...) décrivaient de façon vivante la conquête de la terre : les canaux receuillaient les eaux, la terre émergée se souvenait d'avoir été détrempée depuis deux-mille-cinq-cents ans, et tout le long des canaux, on voyait l'eau s'écouler comme depuis un versant montagneux en miniature. Entre les rencontres et les croisements des canaux, partout, ce doux bruit d'eaux courantes qui donnaient à la plaine un mouvement vital, comme une bonne circulation sanguine. Ici les rigoles, là les grands ruisseaux et les canaux, les anciens avec leur fond vert (...), et ceux récents, faits hier, ou en train de se faire et que les ouvriers creusent sous la pluie, et qui se transforment en ruisseaux troubles à peine ils sont ouverts (Alvaro, 2008:19-20).

Le "doux bruit" de l'eau vive qui s'écoule dans les canaux s'oppose de manière implicite à l'immobilité du marais. L'hydrographie de la région pontine est ici comparable à la vitalité d'une "bonne circulation sanguine" : une autre image qui rappelle, par opposition, la représentation mortifère de l'eau stagnante. Parallèlement au thème des eaux "vivifiées" par les interventions hydrauliques, nous assistons à l'introduction des tournures de la "guerre au marais" : une bataille contre les forces adverses

LE GLOBE - TOME 156 - 2016 
de la nature, condamnées à se plier au régime. Cette interprétation du paysage de bonification à construire est véhiculée par une abondance d'images renvoyant au champ sémantique militaire et à l'idée de conquête. Par exemple :

La lutte avec l'eau devenait un fait vivant, et dans les habitations son long grondement faisait penser à la lutte contre un ennemi. La terre, en la regardant sous son bord, semblait s'ancrer avec ses maisons et ses villages après avoir navigué sur l'abysse boueux, et les maisons coloniales ordonnées à perte de vue semblaient se tenir en rang pour s'encourager. La conquête de l'homme sur l'eau prenait ces jours une valeur définitive, comme si elle subissait cette épreuve avec volupté (Alvaro, 2008:19-20).

Ou encore (Alvaro, 2008:21) :

La terre est bouleversée comme à l'approche d'une guerre.

L'image des nouvelles maisons rurales bâties en mortier les unes derrière les autres, qui "avancent comme une armée en ordre" (Alvaro, 2008:21) est récurrente dans la rhétorique de la "construction des terres nouvelles". La bonification est perçue comme une œuvre qui "crée" un nouveau paysage, ne permettant pas aux zones humides précédentes de prétendre même au titre de paysage. La connotation démiurgique attribuée au processus de bonification mène Alvaro à faire le parallèle entre ses phases initiales et l'épisode biblique de la Genèse. "Parmi les jours de la Création, celui de la séparation de la terre et des eaux devait être le plus beau, car ainsi commença la vie" (Alvaro, 2008:19).

Cette victoire sur les formes sinueuses et variables des zones humides trouve son expression dans les mailles régulières et géométriques du nouveau paysage de bonification :

(...) les canaux et les routes entrelacés, coupent de façon régulière cette vaste zone et donnent l'impression géométrique d'un échiquier. Chacune de ces routes se noue à un bourg qui s'ouvre sur une place (Alvaro, 2008:38).

L'aspect régulier et géométrique du projet hydraulique et de son aménagement suscite de l'admiration pour l'ordre et la discipline. Par exemple, Alvaro écrit que l'emplacement actuel de Sabaudia est rejoint par "une très belle route droite" (Alvaro, 2008:49) ; et il réaffirme plus loin le tracé rectiligne et la netteté des routes, qui représentent 
métaphoriquement l'ordre et la modernité : "(..) le long de la route blanche et droite, aux croisements d'autres routes blanches et droites" (Alvaro, 2008:37).

Pour sa part, Emil Ludwig, dans ses Colloqui con Mussolini (Entretiens avec Mussolini), conversations librement transcrites datant de 1932, affirme avoir survolé la zone où la bonification pontine s'effectuait :

(...) s'étendait sous mes yeux comme sur une carte géographique : je voyais les rangées parallèles des nouveaux sillons dans les champs, la ligne des canaux principaux et secondaires qui portaient à la mer l'eau des marais (Ludwig, 1965:164-165).

On y trouve les rangées parallèles des sillons dans les champs, les lignes des canaux : des droites qui produisent une similitude non due au hasard avec une carte géographique ${ }^{3}$. La bonification apparait comme la volonté de représenter le territoire à l'image d'une carte géographique (Cavallo, 2011). Un espace géométrique, "plat", fortement homogène, dont les grands axes (routiers et hydrographiques) sont pratiquement abstraits, semblent ne pas tenir compte ou être indifférentes au substrat de l'environnement et sont orientées de façon homogène, dans un dessin qui révèle davantage sa cohérence moderne si nous l'observons depuis le ciel, ou encore, depuis l'avion, exactement comme les peintures consacrées à la bonification des marais pontins typiques de l'aéropeinture futuriste ${ }^{4}$.

L'éloge de la modernité de la bonification, et par conséquent du fascisme, est repris dans de nombreuses descriptions et mémoires qui viennent d'une certaine façon du modèle de Terra Nuova d'Alvaro. Cependant, même en mythifiant le paysage de la bonification, Alvaro luimême ne cache pas son admiration pour ce qu'il y avait avant la bonification, royaume des forêts hygrophiles, du vent et des animaux sauvages : "une époque superbe, abandonnée des hommes, en proie aux éléments aveugles et sans gouvernance" (Alvaro, 2008:14) ; même lorsqu'il décrit les quelques habitants des marais ${ }^{5}$ comme des animaux primitifs, il ne dissimule pas son intérêt pour le mode de vie qu'ils représentent, et qui est condamné à disparaître :

Aux limites de la bonification... les derniers de cette vieille humanité qui cerchait dans le marais les poissons et le gibier. Le souvenir de cette vie

LE GLOBE - TOME 156 - 2016 
ancienne se trouve encore partout, comme un chien égaré, produisant un curieux contraste (Alvaro, 2008:20).

Et plus loin : "(...) bergers éternels. Ils regardent la plaine, les maisons bleues qui marquent les étapes de la conquête, la géométrie des canaux" (Alvaro, 2008:17). Et aussi : "Un jour, dans un musée de l'un de ces nouveaux villages, il serait juste de reconstruire une de ces cabanes qui ne seront plus dans quelques jours qu'un souvenir" (Alvaro, 2008:17). Le ton laisse transparaître quelque chose de plus que de la curiosité ethnographique envers ces "sauvages de chez nous", amenant le narrateur à conseiller la muséification des lestre (cabanes en roseaux dans lesquelles vivaient les habitants des marais pontins). En revanche, concernant Romano Pascutto, poète de San Stino di Livenza, dans l'arrière-pays vénitien à la limite avec le Frioul, nous pouvons franchement parler de nostalgie et de regret pour la perte de formes hydrographiques naturelles, attribuée aux méthodes modernes de contrôle des eaux, même quand la bonification n'en est pas responsable. A titre d'exemple, la poésie composée en dialecte de San Stino, I fosseti (Pascutto, 2010:57) :

\author{
où sont-elles les rigoles \\ à l'eau claire entre les feuilles \\ les libellules qui perçaient de leur son \\ l'ombre des branches de robinier? \\ Pleines quand fondait la neige \\ puis un jour à nouveau sèches \\ avec les violettes jaunes par essaims \\ et le désir fou d'y balancer \\ dedans les filles courbées sur elles pour les cueillir \\ Maintenant avec les canaux tout droits \\ Personne n'attend plus les violettes \\ Et pas même les timides jeunes filles
}

Si d'après Pascutto les canaux "si droits" ont effacé l'aspect poétique des fossés, ornés de libellules, violettes et demoiselles, Alvaro ne cède pas à la nostalgie du passé. Au contraire, il considère fondamentale l'antithèse entre 1'aspect primitif inactif des "macchiaroli" (Alvaro, 2008:21) et l'activité moderne aussi bien du fascisme, au niveau politique, que des ingénieurs hydrauliciens qui le représentent, au niveau technique et conceptuel. D'après le régime, la bonification symbolise la modernité sous 
forme de campagne (Cavallo, 2011). La modernité se réfère à la mécanisation ${ }^{6}$ et à la rapidité de transformation du territoire : "Cela n'est jamais arrivé de voir en si peu de temps, et de jour en jour, une aussi ample et complète transformation de la terre, ou peut-être dans l'histoire moderne, dans le phénomène de la guerre (encore une comparaison militaire) (...) les temps se sont raccourcis comme dans un film en mode accéléré" (Alvaro, 2008:38-39).

La transformation des marais pontins peut être assignée à l'aspect "social" du fascisme, étant donné que ce régime avait pour philosophie de racheter des exploitations agricoles et de les attribuer aux anciens combattants. Cependant, la modernité, en termes d'efficacité et de productivité, que les acteurs publics ou privés de la bonification convoitent, risque souvent de dissimuler une certaine avidité pour les terres (et l'argent), au point d'oublier complètement le fait que les véritables éléments qui transforment la campagne sont le climat et le vaet-vient des saisons. Cette "faim de terre" est incarnée par le personnage de Riccardo Tonello, un être sordide et arriviste de La lodola mattiniera, roman de Pascutto de 1977 :

Pour lui, le marais était déjà une terre sèche qui s'étendait graduellement tout autour, jusqu'à l'infini. Il caressait des projets grandioses. (...) Il deviendrait le plus grand propriétaire de la province, peut-être lui auraientils donné le titre de Comte. (...) Il avait récupéré et cousu ensemble, en les achetant à de pauvres gens, beaucoup de petites parcelles près des principaux ouvrages prévus par le projet une fois exécuté, que très peu de gens avaient eu la possibilité de connaître d'avance. Des routes, des canaux, des ponts. Au coeur de la vaste zone bonifiée se serait dressé l'empire des Tonello, projeté vers l'Adriatique" (Pascutto, 1977:157-158).

\section{Bonification : paysage avec machines}

En général, les machines sont une métonymie représentative de la modernité du paysage de bonification, aussi bien celles que l'on utilise pour les travaux d'installations hydrauliques que celles employées pour les travaux à la campagne.

Le roman Canal Mussolini d'Antonio Pennacchi, paru en 2010, fait état de ces machines de manière très précise, aussi bien dans la technique que dans la documentation (Pennacchi, 2010:144) :

LE GLOBE - TOME 156 - 2016 
(...) quatre excavateurs Tosi au travail, plus deux Ruston et un Bucjrus à benne. (...) C'étaient des machines énormes et très longues, qui ressemblaient à des dragons - et en effet elles s'appellent aussi dragues comme des mille-pattes géants : une série de bennes, attachées à un long treillis incliné qui les faisait tourner l'une après l'autre grâce à une chaîne.

D'après Alvaro, les machines agricoles "sont parquées dans les enclos comme des troupeaux d'un nouveau genre" (Alvaro, 2008:21), alors que les équipements utilisés pour le dragage et le déboisement déchirent le silence des marais pontins avec leur "hurlement" (Alvaro, 2008:16). Ainsi, le paysage de bonification est aussi désigné comme soundscape mécanique. Cet élément est également repris, entre autres, par Pietro Ghizzardi, narrateur et peintre naïf qui a vécu entre les paysages bonifiés de Mantoue et d'Emilie-Romagne, dans son autobiographie Mi richordo anchora (Ghizzardi, 1967:220-221) :

Et je passais toujours ma vie très solitaire, et de temps en temps j'allais voir si l'eau était en train de baisser ; elle coulait très lentement, alors les gens de la bonification avaient pensé placer une turbine pour enlever l'eau plus vite, et en effet tous les matins quand j'étais au lit j'entendais le bruit de cette machine qui continuait jour et nuit à turbiner.

Tel que cela apparaît dans le discours indirect libre reproduisant un italien calqué sur le dialecte, les machines de la bonification restent des objets à peine déchiffrables, excepté par la caste des initiés, des techniciens et des ingénieurs hydrauliciens ("ceux de la bonification"). La masse paysanne n'a le droit que d'en écouter les bruits et d'en observer l'aspect extérieur. Outre les pelleteuses, dragues et turbines mobiles, il y avait surtout les pompes de drainage fixes, ces machines fondamentales de la bonification qui détiennent aussi le monopole presque exclusif de sa représentation. Les stations de drainage (ou usines de drainage) qui abritent les pompes n'ont pas encore été mentionnées jusqu'à présent, mais elles constituent un monument incontournable du paysage des "terres nouvelles". Bien au-delà de leur fonction technique, ces constructions imposantes assument une importance symbolique, à l'instar de véritables "cathédrales de la bonification".

Il était impossible aux artistes futuristes de résister au charme des stations de drainage, qui constituaient le noyau mécanique du système 
hydrographique de bonification. Les thèmes de la rapidité et de la machine, chers à la poésie futuriste, trouvent ainsi une dimension rurale insolite. Voici les mots (rigoureusement privés de ponctuation, comme l'exigeaient les normes futuristes) de Filippo Tommaso Marinetti, intitulés Estrazione sistematica di nuovi splendori e nuove musiche dai tecnicismi (Extraction systématique de nouvelles splendeurs et nouvelles musiques des technicismes), rédigés en 1940 pour introduire le poème Poema non umano dei tecnicismi (Marinetti, 2007:236) :

Après avoir bu en vitesse des vrombissements et des bruits sourds des moteurs à explosion des batteries de pompes à eau et motoculteurs hors du cauchemard des marais Pontins abolis immense prodige géométrique et polychrome de moissons nouveaux-nées saturées de soleil et brillants canaux qui transpercent l'horizon...

Revenant à Pennacchi, ce dernier place l'origine des Peruzzi à Ca' Bragadin, calqué sur la région du Polesine de Ca' Vendramin, dominée par la station de drainage du même nom. De plus, en évoquant les histoires de la bonification des plaines situées entre Mesa et Terracina, l'auteur cite l'installation imposante de Mazzocchio et en dessine l'image classique avec le bâtiment de la station de drainage fermant le canal :

Au Quartaccio en effet - à Forcellata - les privés avaient déjà installé la première pompe à eau à vapeur en 1907. Le fascisme, avec l'électricité, les mit partout - à Mazzocchio ils en firent une énorme avec six pompes verticales à hélice, la plus grande d'Europe - et elle, maintenant sur l'Appia et le long de la côte, adossée à la dernière dune, elle voit à la fin de chaque canal ces maisonnées jaunes avec leurs grandes et hautes fenêtres rectangulaires et les pompes à l'intérieur, qui soulèvent l'eau à l'embouchure et la reversent dans la mer (Pennacchi, 2010:141).

Ainsi, après avoir suscité l'admiration sur le courant futuriste, les stations de drainage provoquent de nouveau de l'intérêt, également en matière "d'archéologie hydraulique". Certains écrivains en redécouvrent la nature "mécanique" (macchinica), surtout si elle est comparée aux systèmes traditionnels de contrôle des eaux. C'est le cas de " $\Delta$ " - le titre renvoie au Delta du Pô - un récit de Dario Voltolini (2004) paru récemment, introduit dans un projet collectif consacré aux paysages italiens. Voici les mots consacrés aux stations de drainage :

LE GLOBE - TOME 156 - 2016 
Ils sont perdus, coincés entre les vallées comme des artefacts métalliques. Ils apparaissent comme les membres d'un crustacé spatial, articulés par des jointures boulonnées, comme des mains, ils saisissent avec leurs griffes le terrain. Qu'étreignent-ils ? Ils semblent vouloir extirper un cable, une racine. Ce sont les tuyauteries creuses avec lesquelles la station de pompage pêche dans le monde liquéfié, ce sont les éléments de l'opération de drainage : monde délié, désormais intubé, asséché, bonifié, cultivé, protégé de la menace. (...) Ramasse un galet et lance-le au loin. C'est le son métallique de la tuyauterie de la pompe d'assèchement qui te revient. Mais dans ce monde humide, qu'est-ce qu'elle pêche, extrait ou décharge et pourquoi ? A la différence de la roue hydraulique qui exhibait son travail (la procession des récipients attachés les uns aux autres en enchaînement continuel), la pompe de drainage suce mystérieusement. Venant de l'espace extérieur elle nous colonise, elle se nourrit et grandit en plongeant ses griffes dans le monde acqueux, furibonde créature hydroponique et aliène.

et plus loin :

Maintenant, tout ce travail hydraulique et mécanique qui nécéssitait des briques, des planches en bois et quelques outils (verrous, ferronneries, charnières, leviers, roues) est l'apanage d'un legs extraterrestre qui s'agrippe au terrain et qui pompe, c'est la pompe d'assèchement, la pompe de drainage, qui ne sont pas de ce monde. Et il faut ici encore ce déclic, ce coup sec de l'intuition qui réussit à voir l'alien dans le quotidien. Le jeu des eaux est le même, l'organisation est toujours défensive, pour protéger la terre, qui n'existait pas autrefois, du retour de la mer, de la régurgitation du fleuve. Mais la danse des portes des écluses, le calme de la brique perdue au milieu de la plaine, le théâtre sur l'eau ont été asséchés à la source par l'inextinguible soif vorace de la pompe de drainage : elle semble aspirer même la ligne d'horizon (Voltolini, 2004:15, 28).

\section{Bonification, paysage avec Hommes}

Les citations contenues dans le paragraphe précédent témoignent d'un certain "renouveau" des stations de drainage. Par ailleurs, après un demisiècle passé dans l'oubli total, et grâce à l'association bonification fascisme, les paysages des "terres nouvelles" - plus si nouvelles que cela désormais - refont surface dans le paysage littéraire italien grâce au célèbre roman Canal Mussolini ${ }^{7}$ d'Antonio Pennacchi (2010) ${ }^{8}$.

Bien que le texte ait été déjà cité précédemment, sa présentation nous semble judicieuse. Pennacchi, lui-même descendant de colons de la 
Vénétie installés dans la zone des marais pontins, raconte l'histoire de la famille nombreuse "vénéto-pontine" des Peruzzi, pendant un arc temporel allant des années 1910 à la Seconde Guerre Mondiale. Les Peruzzi, fermiers et métayers de la plaine du Pô (la situation géographique reste volontairement imprécise, mais les références indiquent une zone située entre la Polésine de Rovigo et de Ferrara ${ }^{9}$ ), très tôt sympathisants du fascisme, ont été licenciés dans les années 1920 par les comtes pour lesquels ils travaillaient en métayage. C'est alors qu'ils se tournent vers le gouvernement de Mussolini qui leur propose l'assignation d'une exploitation agricole à vendre et en cours de bonification dans la région pontine : en qualité d'ancien combattant de la Première Guerre Mondiale, le chef de famille, Pericle, y a droit.

"Les marais Pontins ?" a dit oncle Pericle terrorisé. Parce que lui il les avait vus ces marais lorsqu'il était à l'armée - même si c'était du dehors, de loin de Cisterna. Et déjà là se ressentaient les forêts impénétrables, les marais, les étangs et les gens à l'abdomen gonflé comme un ballon. (...) c'étaient les terres de la mort. (...) "Mais non Peruzzi, qu'est-ce que tu as compris ? Nous en avons fait un paradis, nous avons tout asséché, ce n'est plus comme autrefois (...). C'est devenu un jardin, le Jardin Terrestre, et nous donnerons la terre aux paysans" (Pennacchi, 2010:126-127).

Les zones humides sont encore une fois synonymes de paysages impénétrables et mortifères, comme en témoigne la réaction de Pericle. En revanche, concernant le paysage de bonification, la prose salace de Pennacchi, ainsi que le recours important aux discours direct et indirect libre et aux expressions familières et dialectales de la Vénétie ou du Latium, offrent une vision inédite du sujet. En effet, l'auteur louvoie entre le registre sarcastique par rapport à la rhétorique du régime et celui de l'adhésion des colons des marais pontins au ruralisme fasciste : une adhésion sincère mais pas pour autant un aveuglement borné.

Nous sommes clairement face à un exemple de la manière dont un écrivain, pour citer Westphal (2007), est simultanément confronté à un lieu et à sa mémoire littéraire. Si le paysage symbolise l'expression de la mémoire, individuelle et collective (Schama, 1995), le paysage de bonification chez Pennacchi, reconstitué par le biais du filtre des mémoires familiales des Peruzzi, devient l'occasion de se confronter à nouveau non seulement au fascisme en soi (sujet complexe et, par ailleurs,

LE GLOBE - TOME 156 - 2016 
souvent évité en Italie) mais surtout à son héritage territorial. En soutenant les canons fascistes, les caractères et les actions des Peruzzi mettent en évidence certains attraits comme la jeunesse, la fécondité, la force physique, la virilité et les activités militaires. Cependant, l'originalité et la sensibilité des personnages donnent une image de soutien, mais aussi désenchantée et ironique sur "l'épopée pontine".

La première reconstitution du paysage de bonification est décrite par la vision des Peruzzi, tout juste arrivés dans les marais pontins :

(...) Nous arrivâmes lorsque Piscinara était déjà asséchée. Une table rase. Un tapis de billard. Même pas un arbre à l'horizon, de tous ces bois et toutes ces forêts qui étaient là autrefois, d'après oncle Pericle, pullulantes de bêtes, de brigands assassins... Plus une seule goutte d'eau, plus un brin d'herbe, et nous étions trente mille à venir peupler comme des petites quilles ce tapis de billard, un vide sans fin, tout sec, une terre vierge. Cela ressemblait au désert (...) (Pennacchi, 2010:137).

Cette zone de plaines n'est étrangère ni aux Peruzzi ni à leurs compagnons de voyage provenant de Zero Branco (province de Trévise). Néanmoins, sur leurs terres d'origine...

(...) l'horizon n'était pas un vide infini. Il y avait des arbres à Zero Branco, des arbres partout, qui séparaient les champs et les chemins : ormes, acacias, peupliers et frênes gigantesques, qui bordaient les routes et les canaux. Et combien de clochers ! Chaque village avait le sien (...) (Pennacchi, 2010:137-138).

Alors que dans la bonification des marais tout juste achevée...

(...) plus un arbre, rien, tout était dépouillé, d'ici à l'horizon : le vide absolu. Seulement cette bande bleu clair à l'est - les montagnes - et les berges du Canale Mussolini. Berges pourtant nues - terre meuble, sans un brin d'herbe - pas une berge, mais un tumulus, une tombe fraîche sans même une croix. Et des maisonnettes dans la plaine - bleu clair comme les montagnes - mais vides, sans vie, personne à l'intérieur, pas un arbre à côté. (...) Toute une plaine de boue, terre meuble, pas une trace de verdure, d'ivraie ou chiendent ; un désert, un désert de boue (Pennachi, 2010:138139). 
Ici, le "désert" ne se réfère pas aux marais inhabitables, mais - contre toute attente - à la bonification (voici un exemple de retournement de la rhétorique fasciste ${ }^{10}$ ). Il s'agit d'une bonification en voie d'aboutissement d'un point de vue hydraulique (même si, à bien y regarder, elle est encore synonyme de "boue"), mais vierge en ce qui concerne l'anthropisation du paysage. C'est donc le sens du "vide", humain plus que spatial, qui effraie et désoriente les nouveaux arrivés : le paysage de la bonification se retrouve au gué symbolique entre une topophobie (Tuan, 1974) découlant de l'incapacité à reconnaître et attribuer un sens aux lieux, et la construction d'un rapport de reconnaissance mutuelle et d'enracinement à ces derniers. Dans le vide conceptuel d'une terre plate "comme une table de billard" et pauvre en éléments verticaux, les colons cherchent des points d'attache pour se repérer et trouvent les monuments classiques de la bonification : canaux, exploitations agricoles, eucalyptus. ${ }^{11}$

Il s'agit des éléments qui finissent par devenir l'attrait caractérisant du "vide" de chaque plaine bonifiée, notamment au $\mathrm{XX}^{\mathrm{e}}$ s. Une sorte de koinè (Pennacchi, 2008) partagée par des paysages géographiquement distants entre eux (les plaines sardes, les basses plaines vénéto-frioulanes, du Latium - précisément -, mais aussi de nombreux endroits de l'Italie méridionale). ${ }^{12}$

Si le paysage de la bonification est caractérisé par un premier niveau de "vide" qui désigne le visuel, c'est le deuxième niveau qui intéresse particulièrement Pennacchi : le "vide humain". Grâce à l'histoire des Peruzzi, Pennacchi transmet au paysage de la bonification le processus d'humanisation, d'enracinement. Dans cette analyse, les marais pontins ne sont pas construits par les canaux, les exploitations agricoles, les routes, les villages ruraux et les villes nouvelles, mais bien par les colons, dans une représentation comparable à une sorte de laboratoire du lieu de vie.

Alvaro avait déjà évoqué l'aspect humain des premières étapes de peuplement :

Le soir de notre arrivée, et pour quelques jours encore, nous suivimes la nouvelle vie des colons. Occuper la maison, la meubler, reconnaitre la terre (...). Les premières vingt-quatre heures de vie d'un lambeau de terre nouvelle (Alvaro, 2008:37).

L'épopée du peuplement interne est un thème mis en évidence par le fascisme qui proposait de créer un "Homme nouveau" sur les terres

LE GLOBE - TOME 156 - 2016 
nouvelles. La ressemblance de la bonification avec le Far West italien, façonné sur le modèle de l'imaginaire de la conquête américaine, est récurrente. Cela peut sembler paradoxal vu l'antiaméricanisme de Mussolini, mais il est judicieux de rappeler que le fascisme et l'ère rooseveltienne avaient de nombreux points communs concernant la modernisation territoriale interne. ${ }^{13}$ Alvaro, une fois de plus, utilise ces termes pour décrire l'arrivée des colons :

Les familles étaient transportées par des chars tirés par des boeufs. On peut revoir cette scène dans certaines fotos : les chars sont pleins de personnes debout, et on devine les têtes des enfants dépassant à peine des côtés du char, (...) quelques hommes à pied avec des baluchons et des valises (...). C'est une scène comme on en a vu dans des films du Far West (Alvaro, 2008:22-23).

Pier Paolo Pasolini s'approprie lui aussi l'image du Far West (et également celle de la carte géographique, comme nous l'avons évoqué), lorsque, dans Amado mio (1948), il donne forme aux souvenirs des randonnées champêtres juvéniles dans les paysages de bonification des basses plaines de la Vénétie orientale :

Caorle se trouve près de l'embouchure de la Livenza, au point le plus au nord de l'arc dessiné par l'Adriatique entre Trieste et Venise. Son arrièrepays est une bande d'une vingtaine de kilomètres, marécageuse et déserte, bonifiée depuis peu d'années ; et elle est toute entrecroisée de canaux, de berges, de digues ; les champs sont immenses et le maïs est vert comme des pastèques. Après Portogruaro on commence à respirer l'odeur de la Bassa, qui s'ouvre sans fin au delà de Concordia. (...) Les routes de la Bassa étaient des droites infinies, qui se croisaient comme dans une carte géographique ; et ces habitations qui étaient dans le Frioul de simples cabanes, étaient ici de grandes fermes, rouges et blanches. C'était comme si on était entré dans un autre monde, dans le Far West ou en Alaska (Pasolini, 2000:179-180).

\section{Conclusions : la revanche de la perception du lieu sur la géométrie de l'espace}

L'analyse effectuée à partir des œuvres étudiées ici révèle la manière dont la représentation littéraire des paysages de la bonification est soumise, tout d'abord, à des processus inévitables de sélection (choix ou omission de certains éléments territoriaux), mais aussi à des processus de "spécification" pour lesquels une multitude de textes contribuent à adapter

LE GLOBE - TOME 156 - 2016 
une sorte de cliché récurrent et fonctionnel pour évoquer différents paysages géographiques réels (selon le cas, les bonifications de la Vénétie, frioulanes, les marais pontins...) et, en même temps, à construire une image unitaire et hautement symbolique du "paysage de bonification italien". Il a été observé que "la littérature d'imagination ne dépeint pas le monde tel qu'il est mais tel qu'il devrait être ou qu'il pourrait être. (...) La littérature se révèle ainsi un enjeu culturel et idéologique, pas seulement à l'échelle individuelle, mais aussi collective" (Lévy, 2006:35). Pour ce qui est de l'image littéraire du paysage de bonification italien, le point de vue idéologique est particulièrement complexe, étant donné que la bonification représentait l'une des interventions centrales de modification du territoire opérées par le régime fasciste et constituait sans doute l'intervention la plus mise en évidence par le mégaphone de la propagande. Comme chaque produit culturel, l'image littéraire d'un paysage n'est pas gravée éternellement, mais elle fait l'objet de remaniements et d'adaptations continus, oscillant entre le renforcement de certains symboles et l'ouverture à de nouvelles attributions de sens. Au cours de la construction progressive de l'image du paysage de la bonification, des débuts de l'époque contemporaine à aujourd'hui, nous pouvons identifier principalement trois phases successives chronologiquement. Cependant, des ensembles thématiques de représentation qui passent outre la répartition en trois phases ont été développés dans les paragraphes précédents (le rôle de l'élément anthropique et mécanique dans la représentation du paysage).

Une première phase (que nous appellerons phase "classique"), représentée par la Feroniade de Vincenzo Monti, constitue une sorte d'étape préliminaire. En effet, le poème peut être considéré comme le point culminant d'une longue tradition de représentations négatives du marais, déclinée aussi bien à un niveau "culte" que populaire (cf. Cavallo, 2014). Mais le cliché dénigrant du marais prépare déjà le terrain à l'éloge de la bonification en tant qu'instrument qui permet justement d'"effacer" les marais. Le paysage de bonification n'est donc pas décrit et connoté explicitement, mais il est systématiquement évoqué par opposition : si le marais représente la barbarie, la bonification apporte avec elle une garantie de civilité et d'harmonie agricole, au point d'évoquer l'âge d'or arcadien aux saveurs classiques.

LE GLOBE - TOME 156 - 2016 
La seconde phase de la construction littéraire du paysage de bonification (phase de modernisation), représentée ici par Terra Nuova de Corrado Alvaro, est non seulement la plus prolifique en termes quantitatifs ${ }^{14}$, mais aussi la plus connotée d'un point de vue idéologique. Il s'agit à n'en pas douter de la phase correspondant à la production littéraire autour de la bonification fortement encouragée par le régime fasciste. L'éloge du paysage de bonification n'est ici pas seulement complètement explicite, mais presque totalisante et souvent grandiloquente. La bonification est représentée comme le triomphe du contrôle des éléments naturels hostiles ("la guerre au marais") et comme une intervention de construction du territoire fulgurante à partir de rien. Le paysage qui, par conséquent, est délimité, devient l'expression aboutie de la "modernité totalitaire" (Gentile, 2008) du fascisme au niveau rural : régularité, artificialité, géométrie, ordre et discipline sont les éléments qui le caractérisent. Le paysage de bonification devient ainsi la formule d'une vision territoriale moderniste et démiurgique, typique des techniciens modernes (ingénieurs hydrauliciens, agronomes, planificateurs). Il s'agit d'une vision inspirée par un projet territorial cohérent et ambitieux, non exempte d'un certain élan paternaliste qui vise à améliorer les conditions de vie rurale, mais, justement, essentiellement totalitaire (même avant et après le fascisme) envers les personnes, les cultures et les éléments environnementaux amphibies "non appropriés" (Cavallo, 2014) ${ }^{15}$.

La modernité comporte également la présence des machines dans l'environnement (Cavallo, 2011) : c'est alors que la bonification devient un "paysage avec machines", au soundscape (Schafer, 1994) mécanique. Les références font appel à la rapidité, à l'efficacité, au potentiel de transformation des machines, typiques du courant futuriste. Cependant, la représentation mécanique du paysage de bonification laisse parfois le champ à une admiration moins affligée et plus ébahie des machines (notamment des stations de drainage) ou encore à une perception énigmatique de ces objets territoriaux.

La troisième phase (phase contemporaine) est représentée par Canale Mussolini d'Antonio Pennacchi. Après des décennies durant lesquelles le paysage de bonification était marginalisé (pas seulement en littérature), les écrivains italiens ${ }^{16}$ sont de nouveau confrontés à des terres qui ne sont plus "nouvelles". 


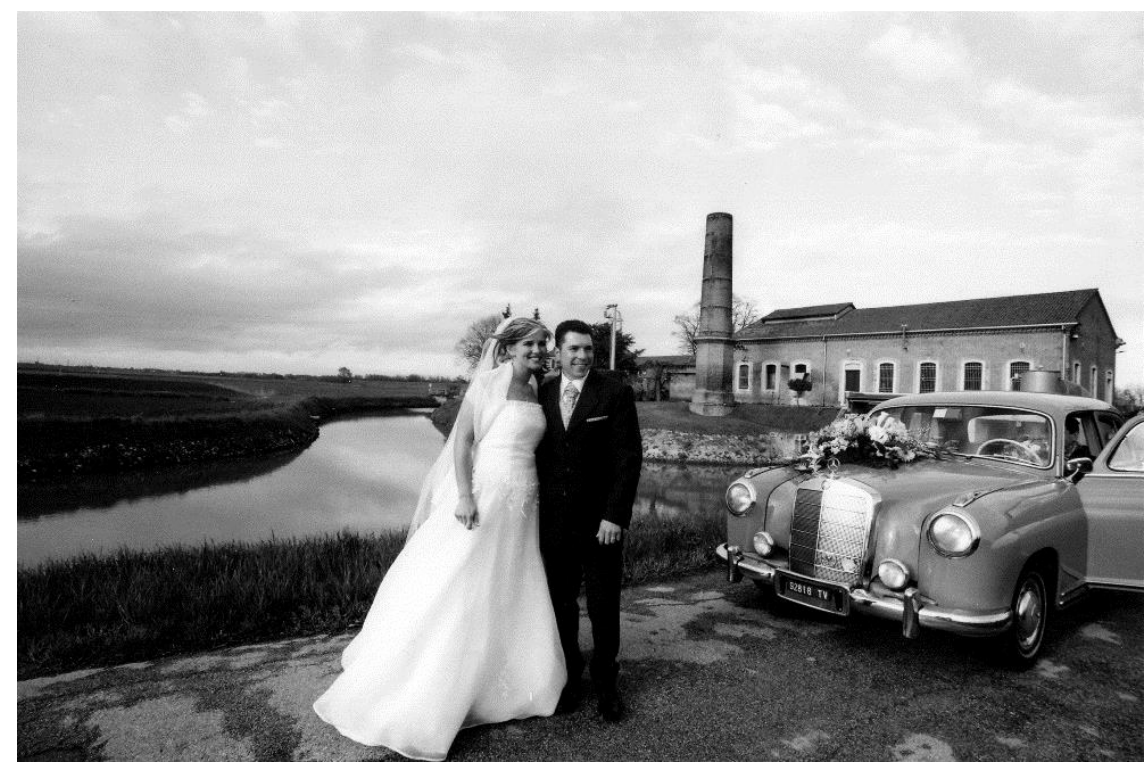

Fig. 1 : L'humanisation contemporaine du paysage de la bonification : jeunes mariés devant la station de pompage de Lova, à Campagna Lupia (Venise) (Photo Damiano Rostellato, 2004)

D'une part, la période mussolinienne est suffisamment éloignée dans le temps pour provoquer un ostracisme implicite sur les paysages de bonification si loués autrefois. D'autre part, le renvoi incontournable à la représentation littéraire renforcée à l'époque fasciste, tout comme sa comparaison avec elle, est évident. La rhétorique élogieuse du régime sert de fond (elle est parfois manifestement démasquée), mais il reste les transformations territoriales vécues par des générations d'hommes et de femmes qui ont contribué à donner un sens, en tant que lieu de vie, aux paysages de la bonification.

L'image du paysage de bonification n'est plus filtrée à travers la vision d'observateurs externes cultivés (tels que Monti e Alvaro), mais sa reconstruction est attribuée à la perception des habitants, notamment des colons appelés à peupler cet espace moderne. Dans cette phase, les vastes horizons ouverts de la bonification caractérisent la représentation du paysage. Celui-ci est dominé par le sens du "vide" : un vide conceptuel,

LE GLOBE - TOME 156 - 2016 
en premier lieu, mais surtout un "vide de vie", dans un contexte où les expériences humaines qui transformeront cet espace en un lieu riche de sens prennent forme. En effet, Alvaro avait déjà évoqué ces sujets, mais il l'avait fait de façon brève : en revanche, dans son roman, Pennacchi peuple le paysage de la bonification d'une humanité variée.

Dans cette optique, l'esthétique du paysage de la bonification évolue également et fait place à une idée d'ouverture liée à la liberté, tout comme dans la fermeture pasolinienne du paysage consacré aux bonifications vénéto-orientales précitées : "Sur ces routes en pierre, sans poussière, on courait divinement à bicyclette - on se sentait légers" (Pasolini, 2000:180).

\section{Bibliographie}

Alvaro C., 2008, Terra Nuova. Prima cronaca dell'Agro Pontino, Milano, Otto/Novecento, éd. orig. 1934.

Baldini E., 1998, Mal'aria, Milano, Feltrinelli.

Brosseau M., 1996, Des romans-géographes, Paris, L'Harmattan.

Cavallo F.L., 2011, Terre, acque, macchine. Geografie della bonifica tra Ottocento e Novecento, Reggio Emilia, Diabasis.

Cavallo F.L., 2014, "Valori geoculturali e turisticità delle zone umide costiere italiane, in : Cavallo F.L. (a cura di), Wetlandia. Tradizioni, valori, turismi nelle zone umide italiane, Padova, Cedam, pp. 1-19.

Chevalier M., 1993, La littérature dans tous ses espaces, Paris, CNRS éditions.

Chevalier M., 2001, Géographie et littérature, Paris, Société de Géographie.

Collot M., 2014, Pour une géographie littéraire, Paris, Editions J. Corti.

De Fanis M., 2001, Geografie letterarie, Roma, Meltemi.

Gentile E. (ed.), 2008, Modernità totalitaria. Il fascismo italiano, Roma, Laterza.

Ghizzardi P., 1981, "Mi richordo anchora" (extrait), in : Guglielmi A. (ed.), Il piacere della letteratura. Prosa italiana dagli anni Settanta ad oggi, Milano, Feltrinelli, pp. 220-223 (éd. orig. 1967).

Lando F., 1993, Fatto e finzione. Geografia e letteratura, Milano, Etaslibri.

Lévy B., 2006, "Géographie et littérature, une synthèse historique", Le Globe, Revue genevoise de géographie, T. 146, pp. 25-52.

LE GLOBE - TOME 156 - 2016 
Ludwig E., 1965, Colloqui con Mussolini, Milano, Mondadori (éd. orig. 1932).

Marinetti F.T., 1940, "Estrazione sistematica di nuovi splendori e nuove musiche dai tecnicismi", in : Sciarretta R. (ed.), Le Parole della Bonifica. Narrativa, poesia, teatro e agro pontino 1922-1942, Latina, Novecento, 2007, pp. 236-238.

Mioni A., 1976, Le trasformazioni territoriali in Italia nella prima età industriale, Venezia, Marsilio.

Monti V., 1839, "La Feroniade", in : Poemetti, Milano, Resnati, pp. 449-548.

Novello E., 2003, La bonifica in Italia. Legislazione, credito e lotta alla malaria dall'Unità al fascismo, Milano, Franco Angeli.

Pascutto R., 1996, Il pretore delle baracche, La lodola mattiniera, Il viaggio, Venezia, Marsilio (éd. orig. 1977).

Pascutto R., 2010, "I fosseti", in : L'acqua, la piera, la tera e altre poesie, Venezia, Marsilio, p 57.

Pasolini P., 2000, Amado mio, Milano, Garzanti, (éd. orig. 1982).

Pennacchi A., 2008, Fascio e Martello. Viaggio per le città del Duce, Roma, Laterza.

Pennacchi A., 2010, Canale Mussolini, Milano, Mondadori.

Pennacchi A., 2015, Canale Mussolini. Parte seconda, Milano, Mondadori.

Pettoello M., 2011, Nelle Terre Basse, Bologna, Bononia University Press.

Pocock D.C.D. (ed.), 1981, Humanistic Geography and Literature. Essays on the Experience of Place, London, Croom Helm.

Schafer R.M., 1994, The soundscape : our sonic environment and the tuning of the world, Rochester (VT), Destiny Books, (éd. orig. 1977).

Sciarretta R. (ed.), 2007, Le Parole della Bonifica. Narrativa, poesia, teatro e agro pontino 1922-1942, Latina, Novecento.

Schivelbusch W., 2008, Tre New Deal. Parallelismi fra gli Stati Uniti di Roosevelt, l'Italia di Mussolini e la Germania di Hitler. 1933-1939, Milano, Marco Tropea Editore, (éd. orig. 2005).

Silone I., 1984, Fontamara, Milano, Mondadori, (éd. orig. 1933).

Stampacchia M., 1983, Tecnocrazia e ruralismo. Alle origini della bonifica fascista (1918-1928), Pisa, ETS.

LE GLOBE - TOME 156 - 2016 
Stampacchia M., 2000, Ruralizzare l'Italia! Agricoltura e bonifiche tra Mussolini e Serpieri (1928-1943), Milano, Franco Angeli.

Voltolini D., 2004, "ه", in : Mozzi G., Voltolini D. (eds), Sotto i cieli d'Italia, Milano, Sironi Editore, pp. 9-35.

Westphal B., 2007, La Géocritique. Réel, fiction, espace, Paris, Les Editions de Minuit.

\section{Notes}

1. Au-delà de l'admiration pour l'œuvre du pape, il est important de signaler que le poète était devenu en 1787 le secrétaire de son neveu, Don Luigi Braschi Onesti, Duc de Nemi, destiné à recevoir une partie considérable des terrains réhabilités sous forme d'emphytéose.

2. A propos du roman Fontamara d'Ignazio Silone, publié en 1933, la référence concerne, quant à elle, la bonification de la plaine du Fucino (Abruzzes) : un cas atypique, non seulement pour le décor mais aussi car il s'agit de l'œuvre d'un auteur antifasciste.

3. Toutefois, nous retrouvons l'utilisation de la même similitude dans Amado mio de Pier Paolo Pasolini.

4. Développé en Italie lors des trois premières décennies du $\mathrm{XX}^{\mathrm{e}}$ s., le futurisme était un mouvement artistique et culturel connu pour sa philosophie basée sur la consécration des canons de la modernité : vitesse, dynamisme, mécanisation... Parmi les principaux représentants figurent les poètes Filippo Tommaso Marinetti et Aldo Palazzeschi et les peintres Umberto Boccioni, Carlo Carrà et Giacomo Balla. L'aéropeinture, en particulier, est un courant futuriste dont la production est inspirée par la vitesse, le dynamisme et la perspective vue du ciel, typique de l'aviation.

5. "Forts de corps, solides, la bouche grande (...) les yeux petits et enfoncés (...) un masque primitif" (Alvaro, 2008:15) ; "générations restées depuis la préhistoire" (Alvaro, 2008:21).

6. On trouvera ci-après, sous Bonification : paysage avec machines une analyse plus approfondie sur le sujet.

7. Il s'agit aussi du nom par lequel le canal principal des marais pontins était baptisé (aujourd'hui Canale delle Acque Alte).

8. Pennacchi a publié plus tard une suite au roman, intitulée Canale Mussolini. Parte seconda (2015).

9. Certains toponymes sont réels : par exemple, Tresigallo (situé près de Ferrara et reconstruite dans les années 30 sous le modèle rationaliste) ; d'autres sont des noms inventés, mais se réfèrent à de vrais lieux (la localité de Ca' Bragadin, déjà

LE GLOBE - TOME 156 - 2016 
mentionnée). De toute façon, les Peruzzi se déplacent d'une zone de bonification à l'autre.

10. De la même façon, concernant le mythe de la rapidité de la transformation territoriale (qui tend à rendre crédible l'idée d'une séparation nette entre un "avant" et un "après" la bonification), Pennacchi écrit : "La bonification ne s'est pas faite du soir au matin. Il a fallu dix ans pour tout assécher et tout aménager, de Cisterna a Terracina, des montagnes à la mer" (Pennacchi, 2010:126).

11. Il s'agit d'une essence exotique acclimatée en bonification grâce à ses besoins considérables en eau (ce qui contribuait au drainage), à son accroissement rapide quand elle servait de brise-vent et aussi car elle pouvait éloigner les moustiques (cf. Pennacchi, 2008).

12. Dans son œuvre Terra Nuova, en vérité, Alvaro avait déjà cueilli d'une certaine manière cette saveur commune à toutes les régions de la bonification, en accentuant leur nature essentiellement rurale. En effet, il terminait sa chronique par : "(...) j'en ressens déjà cette nostalgie, comme d'un village qui comprend tous les villages, nostalgie de celui qui est né près des mottes de terre. (...) Celui qui se souvient des champs d'Italie, et a eu juste pour un instant la fantaisie de s'y arrêter, et voudrait avoir vécu dans la "Bassa" de la Vénétie comme dans les Pouilles, dans la vallée du Pô ou dans la campagne romaine, ici, il ressent tout cela réuni" (Alvaro, 2008:40-41).

13. Il est possible de trouver des références à ce sujet, par exemple dans : Schivelbusch, 2008.

14. Pour un florilège, voir : Sciarretta, 2007.

15. Cependant, nous pouvons voir dans Terra Nova une perspective ethnographique et réfléchie sur le paysage marécageux et sur tout un monde aquatique en voie de disparition : un élément qui semble devancer l'intérêt pour les paysages humides, que l'on retrouve dans l'époque contemporaine.

16. En plus de Pennacchi, consulter aussi Mal'aria d'Eraldo Baldini (2008) et Nelle Terre Basse de Mario Pettoello (2011).

Textes italiens traduits par G. Hochkofler et R. Scariati.

LE GLOBE - TOME 156 - 2016 